TRANSACTIONS OF THE

AMERICAN MATHEMATICAL SOCIETY

Volume 359, Number 2, February 2007, Pages 465-488

S 0002-9947(06)04353-4

Article electronically published on September 12, 2006

\title{
SCROLLAR SYZYGIES OF GENERAL CANONICAL CURVES WITH GENUS $\leq 8$
}

\author{
HANS-CHRISTIAN GRAF V. BOTHMER
}

\begin{abstract}
We prove that for a general canonical curve $C \subset \mathbb{Z}^{g-1}$ of genus $g$, the space of $\left\lceil\frac{g-5}{2}\right\rceil$ th (last) scrollar syzygies is isomorphic to the Brill-Noether locus $C_{\left\lceil\frac{g+2}{2}\right\rceil}^{1}$. Schreyer has conjectured that these scrollar syzygies span the space of all $\left\lceil\frac{g-5}{2}\right\rceil$ th (last) syzygies of $C$. Using Mukai varieties we prove this conjecture for genus 6,7 and 8 .
\end{abstract}

\section{INTRODUCTION}

In this paper we study the syzygies of general canonical curves $C \subset \mathbb{P}^{g-1}$ for $g \leq 8$.

In GL84 Green and Lazarsfeld construct low-rank-syzygies of $C$ from special linear systems on $C$. More precisely linear systems of Clifford index $c$ give a $(g-c-3)$ rd syzygy. We call these syzygies geometric syzygies. Green's conjecture Gre84a paraphrased in this way is

Conjecture (Green). Let $C$ be a canonical curve. Then

$C$ has no geometric pth syzygies $\Longleftrightarrow C$ has no pth syzygies at all.

This conjecture has received a lot of attention in the past few years, and it is now known in many cases [Pet23, Gre84a, Sch86, Sch88, Voi88, Sch91, Ehb94, HR98, Voi02, TiB02, Voi05.

Much less is known about the following natural generalization of Green's conjecture due to Schreyer.

Conjecture (Geometric Syzygy Conjecture). Let $C$ be a canonical curve. Then the geometric pth syzygies span the space of all pth syzygies.

Both conjectures are equivalent for $p \geq \frac{g-3}{2}$ since a general canonical curve has no linear systems of Clifford index $c \leq \frac{g-3}{2}$.

The geometric syzygy conjecture is therefore true, where Green's conjecture is known. Furthermore the case $p=0$ (geometric quadrics) was proved by [AM67] for general canonical curves, and by Gre84b for all canonical curves. The case $p=1$ was done for general canonical curves of genus $g \geq 9$ in $\mathrm{vB} 00$.

In this paper we prove the conjecture in the cases $p=1, g=6,7$ and $p=2$, $g=8$ for general canonical curves. The easiest kind of geometric syzygies are those

Received by the editors November 12, 2002.

2000 Mathematics Subject Classification. Primary 13D02, 14H45, 14C20.

This work was supported by the Schwerpunktprogramm "Global Methods in Complex Geometry" of the Deutsche Forschungs Gemeinschaft and Marie Curie Fellowship HPMT-CT-2001001238.

(C)2006 American Mathematical Society 465

Reverts to public domain 28 years from publication 


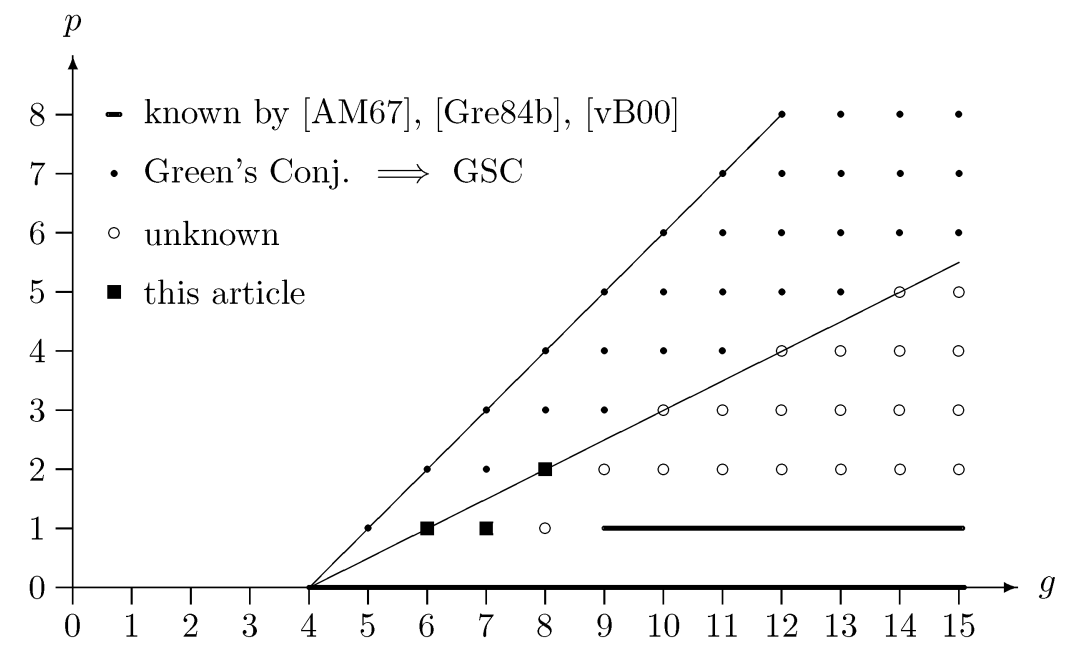

that come from complete pencils $|D|$ of degree $g-d$. By looking at the scroll

$$
S_{|D|}=\bigcup_{D^{\prime} \in|D|} \operatorname{span} D^{\prime}
$$

and its minimal free resolution, one can construct $(d-2)$ nd syzygies of rank $d$. Conversely every $(d-2)$ nd syzygy of rank $d$ determines a scroll whose fibers cut out a linear system of divisors on $C$. We call geometric syzygies of this type scrollar syzygies. To prove the Geometric Syzygy Conjecture in our cases it turns out that it is enough to consider scrollar syzygies. We conjecture that this is always true for general canonical curves.

The starting point of our proof is:

Theorem (Mukai). Every general canonical curve of genus $7 \leq g \leq 9$ is a general linear section of an embedded rational homogeneous variety $M_{g}$. General canonical curves of genus 6 are cut out by a general quadric on a general linear section of a homogeneous variety $M_{6}$.

Using this we first consider the schemes of last minimal rank linear syzygies of the Mukai varieties $M_{6}, M_{7}$ and $M_{8}$ using representation theory. It turns out that all these schemes contain large rational homogeneous varieties.

Passing from Mukai varieties to canonical curves we describe their schemes of last minimal rank syzygies as determinantal loci on the above homogeneous varieties and show that they are scrollar.

Using the resolutions of Eagon-Northcott (for $g=6,8$ ) and Lascoux (for $g=$ 7) we express the cohomology of the corresponding ideal sheafs in terms of the cohomology of homogeneous bundles. The later cohomology is then calculated with the theorem of Bott.

This calculation shows $h^{0}(I(1))=0$, proving the geometric syzygy conjecture in these cases. More precisely our results are:

Theorem A. The scheme $Z$ of last scrollar syzygies of a general canonical curve $C \subset \mathbb{P}^{7}$ of genus 8 is a configuration of 14 skew conics that lie on a 2 -uple embedded $\mathbb{P}^{5} \hookrightarrow \mathbb{P}^{20}$. $Z$ spans the whole $\mathbb{P}^{20}$ of second syzygies of $C$. 
Theorem B. The scheme $Z$ of last scrollar syzygies of a general canonical curve $C \subset \mathbb{P}^{6}$ of genus 7 is a linearly normal ruled surface of degree 84 on a spinor variety $S_{10}^{+} \subset \mathbb{P}^{15}$. This ruled surface spans the whole $\mathbb{P}^{15}$ of first syzygies of $C$.

Theorem C. The scheme $Z$ of last scrollar syzygies of a general canonical curve $C \subset \mathbb{P}^{5}$ of genus 6 is a configuration of 5 skew lines in $\mathbb{P}^{4}$ that spans the whole $\mathbb{P}^{4}$ of first syzygies of $C$.

The paper is organized as follows.

In Section 2 we recall some facts about triple tensors and matrices of linear forms. In particular we introduce the scheme of minimal rank rows $Y_{\min }$ and the vector bundle of linear forms $L_{\text {min }}$ on its reduction $Y_{\text {red }}$. Furthermore we study the restriction of triple tensors.

In Section 3 we define the notion of minimal rank last linear syzygies using the facts about triple tensors from Section 2.

In Section 4 we consider 1-generic triple tensors (matrices of linear forms) and their associated determinantal varieties. In particular we calculate the rank filtration of their last syzygy spaces and determine their reduced scheme of minimal rank last syzygies $Y_{\text {red }}$. In particular $p$ th syzygies of rank $p+2$ can be constructed via scrolls of degree $p+2$.

In Section 5 we prove that conversely every $p$ th syzygy of rank $p+2$ comes from a scroll, and we call these syzygy scrollar. Here we also state a strong form of the scrollar syzygy conjecture for general canonical curves.

Section [6 describes the connection between scrollar syzygies, scrolls and pencils of divisors in the case of a general canonical curve $C \subset \mathbb{P}^{g-1}$. In particular we prove that their reduced spaces of last scrollar syzygies are isomorphic to certain Brill-Noether loci.

Section 7 introduces Mukai varieties and calculates their linear strands. Some large rational homogeneous subspaces of their spaces of minimal rank last syzygies $Y_{\min }$ are identified, and the vector bundle of liner forms $L_{\min }$ on these subspaces is calculated.

In the last three sections we then calculate the spaces of last scrollar syzygies for general canonical curves of genus 8,7 and 6 .

I would like to thank Kristian Ranestad for the many helpful discussions during my stay at Oslo University. It was there where most of the ideas of this work were born.

I dedicate this paper to the memory of my grandmother Lilly-Maria, who introduced me to mathematics.

\section{TRIPLE TENSORS AND MATRICES OF LINEAR FORMS}

Let $A, B$ and $C$ be finite-dimensional vector spaces of dimensions $a, b$ and $c$ together with a map

$$
\gamma: A \otimes B \rightarrow C .
$$

$\gamma$ can be interpreted as a triple tensor $\gamma \in A^{*} \otimes B^{*} \otimes C$ or after choosing bases as an $a \times b$-matrix of linear forms on $\mathbb{P}(C)$.

Definition 2.1. A linear map $\mathbb{C} \rightarrow A$ is called a generalized row of $\gamma$ since it induces a map

$$
\mathbb{C} \otimes B \rightarrow C
$$


which can be interpreted as a $1 \times b$ row vector of linear forms. The the images of such generalized rows $\mathbb{C} \rightarrow A$ form a projective space $\mathbb{P}\left(A^{*}\right)$ which we call the row space of $\gamma$. Similarly $\mathbb{P}\left(B^{*}\right)$ is the column space of $\gamma$.

On the row space $\mathbb{P}\left(A^{*}\right)$ the triple tensor $\gamma$ induces a map of vector bundles

$$
\gamma_{A}: \mathcal{O}_{\mathbb{P}\left(A^{*}\right)}(-1) \otimes B \rightarrow C
$$

by composing it with the first map of the twisted Euler sequence

$$
0 \rightarrow \mathcal{O}_{\mathbb{P}\left(A^{*}\right)}(-1) \otimes B \rightarrow A \otimes B \rightarrow \mathbb{T}_{\mathbb{P}\left(A^{*}\right)}(1) \otimes B \rightarrow 0
$$

on $\mathbb{P}\left(A^{*}\right)$. Similarly we have

$$
\gamma_{B}: A \otimes \mathcal{O}_{\mathbb{P}\left(B^{*}\right)}(-1) \rightarrow C
$$

on the column space $\mathbb{P}\left(B^{*}\right)$. From now on we will restrict our discussion to the row space $\mathbb{P}\left(A^{*}\right)$, leaving the analogous constructions for the column space $\mathbb{P}\left(B^{*}\right)$ to the reader.

Given a generalized row $\alpha \in \mathbb{P}\left(A^{*}\right)$ the restriction of $\gamma_{A}$ to $\alpha$,

$$
\gamma_{\alpha}: B \rightarrow C,
$$

is a map of vector spaces.

Definition 2.2. The rank of a generalized row $\alpha$ is defined as $\operatorname{rank} \alpha:=\operatorname{rank} \gamma_{\alpha}$. The image $\operatorname{Im}\left(\gamma_{\alpha}\right) \subset C$ is called the space of linear forms involved in $\alpha$.

Remark 2.3. The determinantal varieties associated to $\gamma_{A}$ stratify the row space $\mathbb{P}\left(A^{*}\right)$ according to the rank of the rows. In particular the minimal-rank-rows form a closed subscheme $Y_{\min } \subset \mathbb{P}\left(A^{*}\right)$.

Remark 2.4. In practice $Y_{\min }$ is often not of expected codimension, which makes it hard to control as a determinantal variety. In this paper we will construct $Y_{\min }$ via representation theory.

Definition 2.5. On the reduction $Y_{\text {red }}$ of $Y_{\text {min }}$, the image of $\gamma_{A}$ restricted to $Y_{\text {red }}$ is a vector bundle $L_{\min }$ which we call the bundle of linear forms:

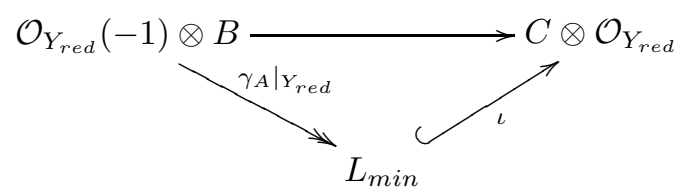

Example 2.6. Consider vector spaces $A, B$ and $C$ of dimension 2, 3 and 4 with basis $a_{i}, b_{i}$ and $c_{i}$. The triple tensor

$$
\gamma: A \otimes B \rightarrow C
$$

with

$$
\begin{array}{lll}
\gamma\left(a_{1} \otimes b_{1}\right)=c_{1}, & \gamma\left(a_{1} \otimes b_{2}\right)=c_{2}, & \gamma\left(a_{1} \otimes b_{3}\right)=c_{3}, \\
\gamma\left(a_{2} \otimes b_{1}\right)=c_{2}, & \gamma\left(a_{2} \otimes b_{2}\right)=c_{3}, & \gamma\left(a_{2} \otimes b_{3}\right)=c_{4},
\end{array}
$$

can be represented by the matrix

$$
\left(\begin{array}{lll}
c_{1} & c_{2} & c_{3} \\
c_{2} & c_{3} & c_{4}
\end{array}\right) .
$$


In this basis we see two rows of rank 3. Generalized rows are linear combinations of those two. The map

$$
\gamma_{A}: \mathcal{O}_{\mathbb{P}\left(A^{*}\right)}(-1) \otimes B \rightarrow C
$$

can be represented by the matrix

$$
\left(\begin{array}{cccc}
a_{1} & a_{2} & 0 & 0 \\
0 & a_{1} & a_{2} & 0 \\
0 & 0 & a_{1} & a_{2}
\end{array}\right)
$$

Since this matrix has full rank everywhere on the row-space $\mathbb{P}\left(A^{*}\right)$ we see that all generalized rows of $\gamma$ have the same rank 3. In particular $Y_{\text {red }}=Y_{\min }=Y_{3}\left(\gamma_{A}\right)=$ $\mathbb{P}\left(A^{*}\right)$. Since $\gamma_{A}$ is injective on $Y_{\min }$ the bundle of linear forms $L_{\min }$ is isomorphic to $\mathcal{O}_{Y_{\min }}(-1) \otimes B$ :

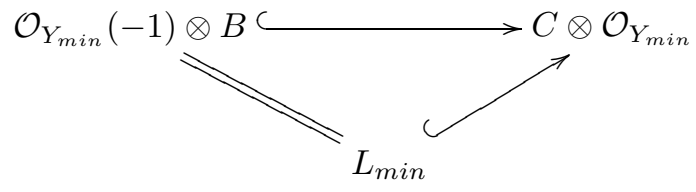

Definition 2.7. A map $\gamma^{\prime}$ obtained by

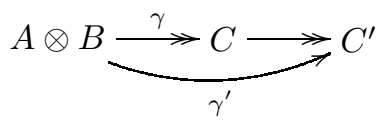

is called a restriction of $\gamma$.

We want to determine how the rank of generalized rows and columns changes during restriction.

Definition 2.8. Consider the composition

$$
\mathcal{O}_{\mathbb{P}\left(A^{*}\right)}(-1) \otimes B \frac{\stackrel{\gamma_{A}}{\longrightarrow} C \otimes \mathcal{O}_{\mathbb{P}\left(A^{*}\right) \longrightarrow}^{\longrightarrow}}{\gamma_{A}^{\prime}} C^{\prime} \otimes \mathcal{O}_{\mathbb{P}\left(A^{*}\right)}
$$

For a generalized row $\alpha \in \mathbb{P}\left(A^{*}\right)$ the rank of the restriction $\gamma_{\alpha}^{\prime}$ is called the restricted rank of $\alpha$.

By definition the restricted rank of a generalized row is always smaller than or equal to its rank.

Remark 2.9. The space $Y_{m i n^{\prime}}^{\prime}$ of minimal restricted rank rows can be completely different from the space $Y_{\min }$ of minimal unrestricted rank rows. Often however $Y_{m i n^{\prime}}^{\prime}$ is a subspace of $Y_{\min }$.

On $Y_{\text {red }}$ we can calculate restricted ranks of minimal rank rows using the vector bundle of linear forms:

Lemma 2.10. Consider the composition

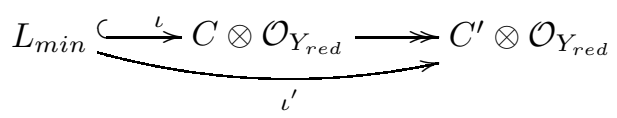

on $Y_{\text {red }}$. If $\alpha \in Y_{\text {min }} \subset \mathbb{P}\left(A^{*}\right)$ is a generalized row of minimal rank, then its restricted rank is the rank of $\left.\iota^{\prime}\right|_{\alpha}$.

Proof. By definition the rank of $\alpha$ is the rank of $\left.\iota\right|_{\alpha}$. By composing with $C \rightarrow C^{\prime}$ we obtain the restricted rank. 
Remark 2.11. If $L_{\text {min }}^{*}$ is globally generated, $C=H^{0}\left(L_{\text {min }}^{*}\right)^{*}$ and $C \rightarrow C^{\prime}$ general, then $\iota^{\prime}$ drops rank in expected codimension. We will use this fact later to describe the restricted rank filtration on $Y_{\min }$ with determinantal varieties. In particular we can calculate the classes of these strata.

Example 2.12. Let $\gamma$ be as in Example 2.6 and $C \rightarrow C^{\prime}$ be the map that sends $c_{4}$ to zero. Then $\gamma^{\prime}$ is represented by

$$
\left(\begin{array}{ccc}
c_{1} & c_{2} & c_{3} \\
c_{2} & c_{3} & 0
\end{array}\right) .
$$

In this basis we see one row that has restricted rank $2 . \gamma_{A}^{\prime}$ is represented by

$$
\left(\begin{array}{ccc}
a_{1} & a_{2} & 0 \\
0 & a_{1} & a_{2} \\
0 & 0 & a_{1}
\end{array}\right)
$$

This matrix drops rank at $(0: 1)$, which corresponds to the bottom row. $Y_{m i n^{\prime}}^{\prime}=$ $Y_{2}\left(\gamma_{A}^{\prime}\right)$ is therefore one triple point in $Y_{\min }=Y_{3}\left(\gamma_{A}\right)=\mathbb{P}\left(A^{*}\right)$.

For the map $C \rightarrow C^{\prime}$ that sends $c_{3}$ to zero $\gamma_{A}^{\prime}$ is represented by

$$
\left(\begin{array}{ccc}
a_{1} & a_{2} & 0 \\
0 & a_{1} & 0 \\
0 & 0 & a_{2}
\end{array}\right)
$$

and we have two rows that drop rank. For a general map $C \rightarrow C^{\prime}$ we expect 3 distinct generalized rows with restricted rank 2 .

\section{LAST LINEAR SYZYGIES}

Let $X \subset \mathbb{P}^{n-1}$ be any projective variety. We denote its minimal free resolution by

$$
F_{\bullet} \rightarrow I_{X} \quad \text { or } \quad F_{\bullet}[1] \rightarrow \mathcal{O}_{\mathbb{P}^{n-1}} \rightarrow \mathcal{O}_{X}
$$

where we consider $F_{\bullet}$ as a bounded chain complex

$$
F_{\bullet}: 0 \rightarrow \cdots \rightarrow F_{2} \rightarrow F_{1} \rightarrow F_{0}
$$

with homology $I_{X}$ concentrated in degree 0 .

If $F_{\bullet}$ is any free chain complex with $F_{i}=\bigoplus_{j} F_{i j} \otimes \mathcal{O}(-i-j)$ and $F_{i j}$ vector spaces, the dimensions

$$
\beta_{i j}=\operatorname{dim} F_{i j}
$$

are called graded Betti numbers of $F_{\bullet}$. Sometimes we will write it more shortly as

$$
F_{i}=\bigoplus_{j} \mathcal{O}(-i-j)^{\beta_{i j}}
$$

or collect the graded Betti numbers $\beta_{i j}$ in a so-called Betti diagram:

\begin{tabular}{c|ccc}
$\beta_{00}$ & $\beta_{10}$ & $\ldots$ \\
$\beta_{01}$ & $\beta_{11}$ & \\
$\vdots$ & & \\
& & $\beta_{i j}$
\end{tabular}

For better readability we will write a dash ("_") if $\beta_{i j}=0$. 
Example 3.1. The rational normal curve $X \subset \mathbb{P}^{3}$ of degree 3 has minimal free resolution

$$
0 \rightarrow \mathcal{O}(-3)^{2} \rightarrow \mathcal{O}(-2)^{3} \rightarrow \mathcal{O} \rightarrow \mathcal{O}_{X} \rightarrow 0
$$

The corresponding Betti diagram is therefore

$$
\begin{array}{ccc}
1 & - & - \\
- & 3 & 2
\end{array}
$$

Note that this notation yields diagrams as calculated by the computer program Macaulay GS02.

To study the minimal free resolution $F_{\bullet} \rightarrow I_{X}$ of a variety $X$ it is often useful to linearize its information. We will look at particular subcomplexes of $F_{\bullet}$ whose differentials can be given as matrices of linear forms.

Definition 3.2. Let $I_{X}$ be an ideal sheaf on $\mathbb{P}^{n-1}$, and

$$
F_{\bullet} \rightarrow I_{X}
$$

a minimal free resolution of $I_{X}$. Let $d$ be the smallest degree, such that $\beta_{0 d} \neq 0$. We define the complex $F_{\bullet}(d)$ by

$$
F_{i}(d)=F_{i d} \otimes \mathcal{O}(-i-d)=\mathcal{O}(-i-d)^{\beta_{i d}}
$$

with the differentials induced from $F_{\bullet}$. We call

$$
F_{\bullet}(d) \rightarrow I_{X}
$$

the degree $d$ linear strand of $I_{X}$, since the differentials of $F_{\bullet}(d)$ are given by matrices of linear forms, and the map to $I_{X}$ is defined by polynomials of degree $d$.

Example 3.3. Let $F_{\bullet} \rightarrow I_{X}$ be the minimal free resolution of the rational normal curve in $\mathbb{P}^{3}$. Since $I_{X}$ is generated by quadrics, its linear strand is $F_{\bullet}(2)$. Its Betti diagram is

$$
\begin{array}{ll}
- & - \\
3 & 2
\end{array}
$$

Remark 3.4. $F_{\bullet}(d) \rightarrow I_{X}$ is a subcomplex of $F_{\bullet} \rightarrow I_{X}$.

Definition 3.5. Let $F_{\bullet} \rightarrow I_{X}$ be the minimal free resolution of $I_{X}$. If $F_{\bullet}=F_{\bullet}(d)$ we say that $I_{X}$ has a linear free resolution of degree $d$.

Remark 3.6. The most important examples of schemes with linear free resolution are maximal determinantal varieties of expected codimension. These are discussed in the next section.

In this paper we apply the discussion of the preceding section to the study of last linear syzygies.

Definition 3.7. Let $C$ be a finite-dimensional $\mathbb{C}$ vector space and $F_{\bullet}(d) \rightarrow I_{X}$ the linear strand of a variety $X \subset \mathbb{P}(C)$. Then the maximal $l$ with $\beta_{l d} \neq 0$ is called the length of the linear strand. The space $\mathbb{P}\left(F_{l d}^{*}\right)$ is called the space of last syzygies of $X$.

We now observe that the last map in the linear strand $F_{\bullet}(d)$,

$$
F_{l d} \otimes \mathcal{O}(-d-l) \stackrel{\phi_{l}}{\longrightarrow} F_{l-1, d} \otimes \mathcal{O}(-d-l+1),
$$

induces a triple tensor

$$
\gamma: F_{l d} \otimes F_{l-1, d}^{*} \rightarrow C
$$


Remark 3.8. Since a last syzygy $s \in F_{l d}$ defines a generalized row $\mathbb{C} \stackrel{s}{\rightarrow} F_{l d}$ of $\gamma$, the row space $\mathbb{P}\left(F_{l d}^{*}\right)$ of $\gamma$ is just the space of last syzygies.

Definition 3.9. The rank of a last syzygy $s \in F_{l d}$ is defined as the rank of the corresponding generalized row $\mathbb{C} \rightarrow F_{l d}$ of $\gamma$. In this setting we will call $Y_{\text {min }} \subset$ $\mathbb{P}\left(F_{l d}^{*}\right)$ the space of last minimal rank syzygies.

\section{1-GENERIC SPACES}

In this section we consider the last syzygies of certain determinantal varieties and describe their ranks.

Definition 4.1 (1-generic spaces). A triple tensor

$$
\Gamma: A \otimes B \rightarrow C
$$

is called 1-generic, if all generalized rows have rank $b$ and all generalized columns have rank $a$. Usually one assumes $a \geq b$.

1-generic triple tensors have many interesting properties. See Eisenbud [Eis88] for a discussion. In particular the induced map

$$
\Gamma_{C}: A \otimes \mathcal{O}_{\mathbb{P}(C)}(-1) \rightarrow B^{*}
$$

on $\mathbb{P}(C)$ drops rank in expected codimension, and this determinantal locus $X=$ $X_{b-1}\left(\Gamma_{C}\right)$ is resolved by the Eagon-Northcott complex

$$
E_{\bullet} \rightarrow I_{X}
$$

with $E_{\bullet}$ linear of degree $b$ and

$$
E_{i}=E_{i b} \otimes \mathcal{O}(-i-b), \quad E_{i b}=\Lambda^{b+i} A \otimes \Lambda^{b} B \otimes S_{i} B .
$$

The maps of $E_{\bullet}$ are induced by triple tensors

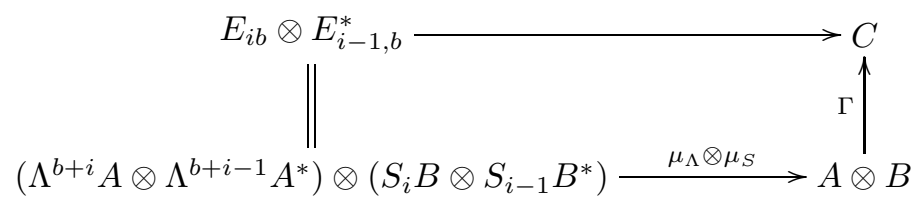

with $\mu_{\Lambda}$ and $\mu_{S}$ the usual contractions.

Lemma 4.2. The space $\mathbb{P}\left(E_{a-b, b}^{*}\right)$ of last syzygies of $X$ is isomorphic to $\mathbb{P}\left(S_{a-b} B^{*}\right)$. The rank filtration of last syzygies is given by

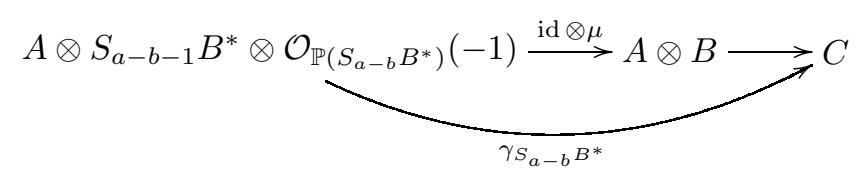

where $\mu$ is induced by

$$
\mu_{S}: S_{a-b-1} B^{*} \otimes S_{a-b} B \rightarrow B .
$$

Proof. A last syzygy $s \in E_{a-b, b}=\Lambda^{a} A \otimes \Lambda^{b} B \otimes S_{a-b} B$ defines a polynomial in $S_{a-b} B$ up to a constant. Therefore $\mathbb{P}\left(E_{a-b, b}^{*}\right) \cong \mathbb{P}\left(S_{a-b} B^{*}\right)$. 
Now the last triple tensor $\gamma$ is given by

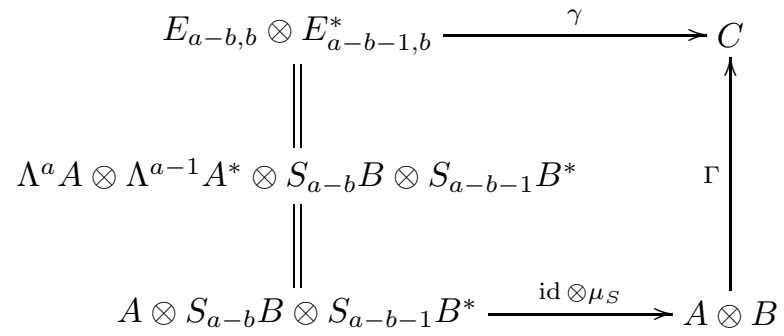

in particular the rank filtration of $\mathbb{P}\left(E_{a-b, b}^{*}\right)$ given by

$$
\gamma_{E_{a-b, b}}: \mathcal{O}_{\mathbb{P}\left(E_{a-b, b}^{*}\right)}(-1) \otimes E_{a-b-1, b}^{*} \rightarrow C
$$

is the same as the rank filtration of $\mathbb{P}\left(S_{a-b} B^{*}\right)$ given by

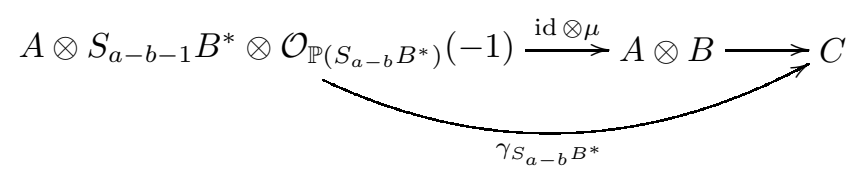

with $\mu$ as claimed.

With this we can describe the space of minimal rank last syzygies in the EagonNorthcott complex:

Proposition 4.3. Let $\Gamma: A \otimes B \rightarrow C$ be 1-generic. Then the reduced space of minimal rank last syzygies of the associated determinantal variety is

$$
Y_{\text {red }} \cong Y_{a} \cong \mathbb{P}^{b-1} \cong \mathbb{P}\left(B^{*}\right) \hookrightarrow \mathbb{P}\left(S_{a-b} B^{*}\right)
$$

with the embedding given by the $(a-b)$-uple map. If $b=2$ all syzygies outside of $Y_{\text {red }}$ have maximal rank.

Proof. Let $s \in S_{a-b} B^{*}$ be a last syzygy. The linear forms involved in $s$ are determined by the image of $\mu$ restricted to $s$,

$$
\mu_{s}: S_{a-b-1} B^{*} \rightarrow B \text {. }
$$

Let $B_{s} \subset B$ be the image of $\mu_{s}$; then rank of $s$ is the rank of

$$
\operatorname{Im}\left(A \otimes B_{s} \rightarrow A \otimes B \rightarrow C\right) .
$$

Since $A \otimes B_{s} \rightarrow C$ is also 1-generic,

$$
\operatorname{rank} s \geq \operatorname{rank} A+\operatorname{rank} B_{s}-1 \geq a
$$

with equality if and only if $\operatorname{rank} B_{s}=1$. This happens only if $s=\beta^{a-b}$ for some $\beta \in B$. This proves the first assertion. If $\operatorname{rank} B=2$ the only other possibility is $B_{s}=B$, and therefore rank $s=\operatorname{rank} \operatorname{Im}(A \otimes B \rightarrow C)$.

Example 4.4. A rational normal scroll $X \subset \mathbb{P}(C)$ of degree $a$ and codimension $a-1$ is cut out by the maximal minors of a 1-generic $a \times 2$-matrix

$$
\Gamma: A \otimes B \rightarrow C .
$$

By the proposition above the last linear syzygies of $X$ have minimal rank $a$, and the space of minimal rank last syzygies

$$
Y_{\text {red }} \cong Y_{a} \cong \mathbb{P}\left(B^{*}\right) \cong \mathbb{P}^{1} \subset \mathbb{P}^{a-2}
$$




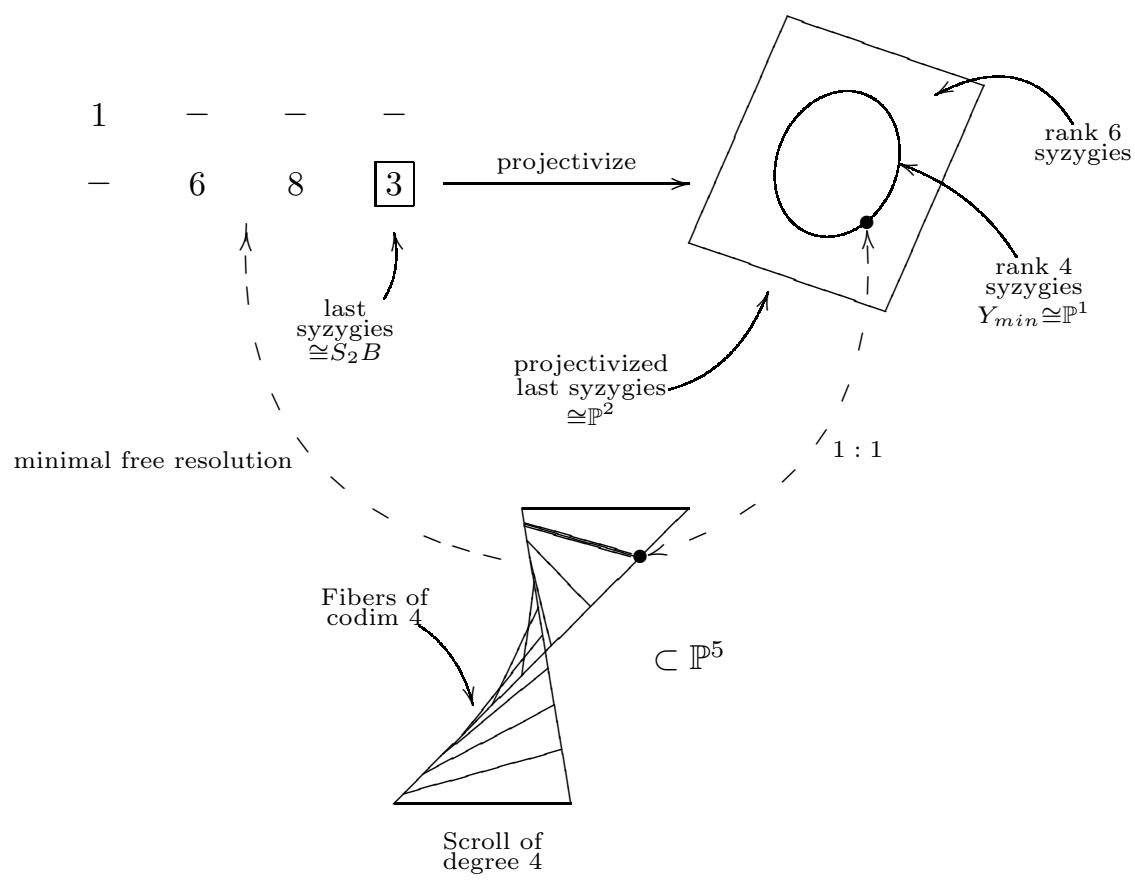

Figure 4.5. Minimal rank last syzygies of a degree 4 rational normal scroll in $\mathbb{P}^{5}$

is a rational normal curve of degree $a-2$. The isomorphisms show that there is a $1: 1$ correspondence between minimal rank last syzygies $s \in Y_{\min }$ generalized columns $\mathbb{C} \rightarrow B$ of $\Gamma$. Since each fiber of $X$ is cut out by the linear forms of a generalized column of $\Gamma$, we also have a $1: 1$ correspondence between minimal rank syzygies and fibers of $X$.

\section{SCROllar SYZYGies}

In Example 4.4 we have seen that every scroll of degree $a$ has $(a-2)$ nd syzygies of rank $a$. In this section we show that in a certain sense this is the only way that such syzygies can arise.

Proposition 5.1. Let $X \subset \mathbb{P}^{n}$ be a non degenerate irreducible scheme with a linear strand of degree 2

$$
F_{\bullet}(2) \rightarrow I_{X}
$$

and let $s \in F_{p 2}$ be a pth syzygy of rank $p+2$. Then there exists the subcomplex

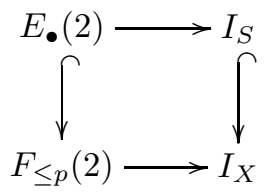

that resolves the ideal sheaf $I_{S}$ of a scroll $S$ of degree $p+2$ and codimension $p+1$ that contains $X$. Furthermore there is a minimal rank last syzygy $s^{\prime} \in E_{p 2}$ of $I_{S}$ that maps to $s \in F_{p 2}$. 
Proof. $s \in F_{p 2}$ induces a diagram

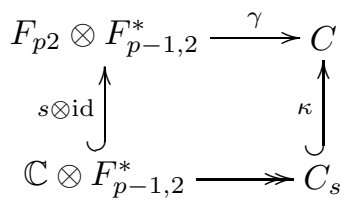

where $\gamma$ is the $p$ th triple tensor of $F_{\bullet}(2)$ and $\operatorname{dim} C_{s}=\operatorname{rank} s=p+2$. Consider now the Koszul complex

$$
K_{\bullet} \rightarrow \Lambda^{l+1} C_{s}^{*} \otimes \mathcal{O}(-1)
$$

with $K_{i}=K_{i 2} \otimes \mathcal{O}(-i-2)$ and $K_{i 2}=\Lambda^{p-i} C_{s}^{*}$ induced by $\kappa^{*}$. The diagram above induces maps

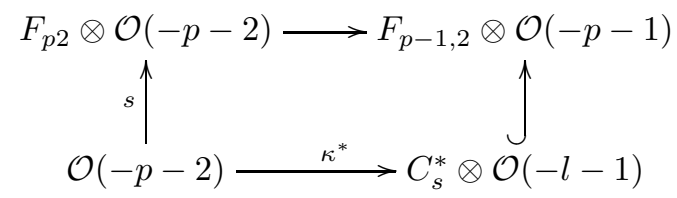

that lift to a map of complexes

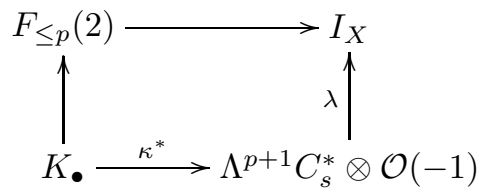

since $F_{\bullet}$ is a complex and $K_{\bullet}$ is exact. The lifting is even unique, since $F_{\bullet}$ and $K_{\bullet}$ are minimal complexes. Since $\kappa$ and $\lambda$ are of degree 1 they can be described by matrices of linear forms $\kappa^{*}=\left(c_{1}, \ldots, c_{l+2}\right)$ and $\lambda=\left(c_{1}^{\prime}, \ldots, c_{l+2}^{\prime}\right)$ after choosing a basis of $C_{s}^{*}$. A straightforward computation shows that the image of $\kappa^{*} \circ \lambda$ is generated by the $2 \times 2$ minors of

$$
M=\left(\begin{array}{cc}
c_{1} & c_{1}^{\prime} \\
\vdots & \vdots \\
c_{p+2} & c_{p+2}^{\prime}
\end{array}\right) .
$$

$M$ is 1-generic. If it was not, we can assume after row and column transformations

$$
M=\left(\begin{array}{cc}
c_{1} & 0 \\
c_{2} & c_{2}^{\prime} \\
\vdots & \vdots
\end{array}\right)
$$

and the first minor degenerates to $c_{1} c_{2}^{\prime}$. This is impossible since $c_{1} c_{2}^{\prime} \in I_{X}$ and $X$ is irreducible and nondegenerate. So the minors of $M$ cut out a scroll $S$ that contains $X$, i.e. $\operatorname{Im}\left(\kappa^{*} \circ \lambda\right)=I_{S}$. The Eagon-Northcott complex $E_{\bullet}(2) \rightarrow I_{S}$ that resolves $S$ is then a subcomplex of $F_{\bullet} \rightarrow I_{X}$. The syzygy $s^{\prime} \in E_{l 2}$ is the minimal rank syzygy associated to the first column of $M$. 
Example 5.2. A general canonical curve of $C$ genus 8 has a minimal free resolution with graded Betti numbers

$$
\begin{array}{ccccccc}
1 & - & - & - & - & - & - \\
- & 15 & 35 & 21 & - & - & - \\
- & - & - & 21 & 35 & 15 & - \\
- & - & - & - & - & - & 1
\end{array}
$$

A second syzygy of rank 4 is an element in 21-dimensional second syzygy space above, that is mapped to a 4-dimensional subspace of the 35-dimensional space of first syzygies:

$$
\begin{array}{cccc}
1 & - & - & - \\
- & 15 & 35 & 21 \\
& & \cup & \cup \\
& & 4 & 1
\end{array}
$$

comparing the Koszul complex of $s$ with the linear stand $C$ yields 6 special quadrics in the ideal of $C$ :

$$
\begin{aligned}
& 1-\quad-\quad- \\
& \text { - } 15 \quad 3521 \\
& \cup \cup \cup \\
& \begin{array}{lllll}
1 & 4 & 6 & 4 & 1
\end{array}
\end{aligned}
$$

In the proof above we have seen that these 6 quadrics are the $2 \times 2$-minors of a $4 \times 2$ matrix with linear entries. Therefore they cut out a scroll of degree 4 that contains $C$. Its minimal free resolution is a Eagon-Northcott subcomplex of the linear strand of $C$ :

$$
\begin{array}{cccc}
1 & - & - & - \\
- & 15 & 35 & 21 \\
\multicolumn{4}{c}{\cup} \\
1 & - & - & - \\
- & 6 & 8 & 3
\end{array}
$$

The above proposition suggest the following definition.

Definition 5.3. Let $I_{X}$ be an ideal sheaf with a linear strand of degree 2

$$
F_{\bullet}(2) \rightarrow I_{X}
$$

of length $l$. A syzygy $s \in F_{p 2}$ of rank $p+2$ is called a scrollar pth syzygy. A last syzygy $l \in F_{l 2}$ of rank $l+2$ is called scrollar last syzygy.

Example 5.4. A rational normal scroll $X \subset \mathbb{P}^{5}$ of degree 4 has a linear strand of length 2 and a conic of last syzygies of rank 4. These minimal rank last syzygies are scrollar (see Figure 4.5).

Remark 5.5. Scrollar syzygies are the easiest examples of the geometric syzygies constructed by Green and Lazarsfeld in GL84.

We can now make a precise statement of the Geometric Syzygy Conjecture for general canonical curves.

Conjecture 5.6 (Scrollar Syzygy Conjecture). Let $C \subset \mathbb{P}^{g-1}$ be a general canonical curve of genus $\mathrm{g}$. Then all minimal rank syzygies are scrollar, and the spaces of scrollar syzygies are nondegenerate. 
Remark 5.7. Equivalently one can conjecture that the scrollar syzygies span the space of all syzygies or that every syzygy of a general canonical curve can be written as a sum of scrollar syzygies. Since, as explained in the next section, scrollar syzygies are closely connected to special pencils on $C$, this would give a geometric interpretation for all syzygies of a general canonical curve.

Remark 5.8. For special canonical curves it is important to consider the nonreduced scheme structure on the space of scrollar syzygies as can be seen in the case of a curve of genus 6 with only one $g_{4}^{1}$ [AH81, p. 174].

Remark 5.9. There are geometric $p$ th syzygies in the sense of Green and Lazarsfeld GL84 which are not of rank $p+2$. These must also be considered in the case of special curves. The easiest example of this phenomenon is exhibited by a plane quintic curve of genus 6 vB00.

\section{Pencils}

We now turn to the connection between scrolls and pencils. Here we restrict ourselves to the case of a canonical curve $C \subset \mathbb{P}^{g-1}$. Let $|D|$ be a complete pencil of degree $d$ on $C$. Then we can consider the union

$$
S_{|D|}=\bigcup_{D^{\prime} \in|D|} \operatorname{span} D^{\prime} \subset \mathbb{P}^{g-1},
$$

where span $D^{\prime}$ is the linear space spanned by $D^{\prime}$ in $\mathbb{P}^{g-1}$.

Proposition 6.1. $S_{|D|}$ is a rational normal scroll of codimension $g-d$ containing $C$.

Proof. Since $C$ is canonically embedded, $\mathbb{P}^{g-1}=\mathbb{P}\left(H^{0}(K)\right)$ with $K$ a canonical divisor on $C$. The set of hyperplanes in $\mathbb{P}^{g-1}$ vanishing on $D^{\prime}$ is therefore

$$
H^{0}\left(K-D^{\prime}\right)=H^{1}\left(D^{\prime}\right)^{*}
$$

and the codimension of span $D^{\prime}$ is correspondingly $h^{1}\left(D^{\prime}\right)=g-d+1$ by RiemannRoch. This is the same for all $D^{\prime} \in|D|$. So $S_{|D|}$ is a rational scroll of codimension $g-d$. Its equations are given by the $2 \times 2$-minors of the $(g-d+1) \times 2$-matrix obtained from the triple tensor

$$
H^{0}(K-D) \otimes H^{0}(D) \rightarrow H^{0}(K) .
$$

$S_{|D|}$ contains $C$ since $D$ moves in a pencil.

Conversely consider a scroll containing $C$. Its fibers cut out a pencil of divisors on $C$. These pencils are not always complete.

Proposition 6.2. Let $C \subset \mathbb{P}^{g-1}$ be a nonhyperelliptic canonical curve of genus $g$ contained in a scroll $S$ of codimension c. Let $F$ be a fiber of $S$ and $D=C . F$. Then $|D|$ is a $g_{d}^{r}$ with $r \geq 1$ and $d \leq g+r-c-1$.

Proof. The fibers of $S$ cut out a pencil of divisors linearly equivalent to $D$. Therefore $r=\operatorname{dim}|D| \geq 1$.

The codimension of a fiber $F$ is $c+1$, so $h^{0}(K-D) \geq c+1$. Riemann-Roch now gives

$$
d=h^{0}(D)-h^{0}(K-D)-1+g \leq r+1-(c+1)-1+g=g+r-c-1 .
$$


In particular these linear systems have low Clifford index.

Corollary 6.3. In the situation above we have cliff $(D) \leq g-c-2$. If the corresponding complete linear system $|D|$ is not a pencil, we even have cliff $(D) \leq g-c-3$.

Proof.

$$
\operatorname{cliff}(D)=d-2 r \leq g-c-1-r \leq \begin{cases}g-c-2 & \text { if } r=1 \\ g-c-3 & \text { if } r>1\end{cases}
$$

Corollary 6.4. A general canonical curve $C \subset \mathbb{P}^{g-1}$ has no pth scrollar syzygies for $p \geq\left\lceil\frac{g-5}{2}\right\rceil$.

Proof. Let $s \in F_{p 2}$ be a $p$ th scrollar syzygy. Then the corresponding scroll $S_{s}$ has codimension $p+1$ by Proposition 5.1. The divisor $D$ cut out by a fiber of $S$ has Clifford index

$$
\operatorname{cliff}(D) \leq g-p-3 .
$$

On the other hand it is well known that on a general canonical curve all divisors have Clifford index at least $\left\lceil\frac{g-2}{2}\right\rceil$. Therefore

$$
p \leq g-3-\left\lceil\frac{g-2}{2}\right\rceil=\left\lceil\frac{g-5}{2}\right\rceil .
$$

Corollary 6.5. The scrollar syzygy conjecture implies Green's conjecture for general canonical curves.

Proof. Assume the scrollar syzygy conjecture. Then every pth syzygy can be written as a sum of $p$ th scrollar syzygies. By the corollary above there are no $p$ th scrollar syzygies for $p>\left\lceil\frac{g-5}{2}\right\rceil$. Therefore there can be no $p$ syzygies at all for $p>\left\lceil\frac{g-5}{2}\right\rceil$. This is Green's conjecture for the general canonical curve.

We now want to consider the last step in the resolution of a general canonical curve that still allows scrollar syzygies.

Definition 6.6. Let $C \subset \mathbb{P}^{g-1}$ be a general canonical curve. Then the scrollar $\left\lceil\frac{g-5}{2}\right\rceil$ th syzygies of $C$ are called the last scrollar syzygies of $C$.

Remark 6.7. Last scrollar syzygies are last syzygies in the sense of Definition 3.7 since Green's conjecture holds for general curves by Voi02] and Voi05.

For the last scrollar syzygies everything is as nice as possible. First we calculate the degree of the corresponding divisors.

Lemma 6.8. Let $C \subset \mathbb{P}^{g-1}$ be a general canonical curve, $s$ a last scrollar syzygy, $S_{s}$ the corresponding scroll and $D_{s}$ the divisor cut out by the fiber $F_{s}$ corresponding to s. Then $\left|D_{s}\right|$ is a complete pencil of degree $\left\lceil\frac{g+2}{2}\right\rceil$.

Proof. Suppose $\left|D_{s}\right|$ was not a complete pencil. Then by Corollary 6.3 we would have

$$
\text { cliff } D_{s} \leq g-\left\lceil\frac{g-5}{2}\right\rceil-4=\left\lceil\frac{g-4}{2}\right\rceil,
$$

which is impossible for a general canonical curve. Consequently we have $r=1$ and cliff $D_{s}=\left\lceil\frac{g-2}{2}\right\rceil$, the minimum possible value. In particular

$$
d=\operatorname{cliff}\left(D_{s}\right)+2 r=\left\lceil\frac{g+2}{2}\right\rceil .
$$


This allows us to construct a morphism from the space of last scrollar syzygies to the corresponding Brill-Noether locus.

Proposition 6.9. Let $C \subset \mathbb{P}^{g-1}$ be a general canonical curve, and $Y_{\text {red }}$ its reduced scheme of last scrollar syzygies. Then there exists an isomorphism

$$
\zeta: Y_{\text {red }} \rightarrow C_{\left\lceil\frac{g+2}{2}\right\rceil}^{1} \text {. }
$$

In particular $Y_{\text {red }}$ is a disjoint union of

$$
\frac{2}{g+2}\left(\begin{array}{c}
g \\
\frac{g}{2}
\end{array}\right)
$$

rational curves if $g$ is even, and an irreducible ruled surface over $W_{\left\lceil\frac{g+2}{2}\right\rceil}^{1}$ if $g$ is odd.

Proof. Consider the vector bundle of linear forms $L$ on the variety of $Y_{\text {red }}$ of last scrollar syzygies. Let $Q$ be the cokernel of the natural inclusion

$$
0 \rightarrow L \rightarrow H^{0}(K) \otimes \mathcal{O}_{Y_{\text {red }}} \rightarrow Q \rightarrow 0 \text {. }
$$

$Q$ is globally generated and has rank $\left\lceil\frac{g}{2}\right\rceil$. It therefore induces a morphism

$$
\begin{aligned}
\alpha: Y_{\text {red }} & \rightarrow \mathbb{G}, \\
s & \rightarrow F_{s},
\end{aligned}
$$

where $\mathbb{G}:=\mathbb{G}\left(H^{0}(K),\left\lceil\frac{g}{2}\right\rceil\right)$ is the Grassmannian of $\left\lceil\frac{g}{2}\right\rceil$-dimensional quotient spaces of $H^{0}(K)$, or equivalently the Grassmannian of $\left\lceil\frac{g-2}{2}\right\rceil$-dimensional linear subspaces of $\mathbb{P}^{g-1} . F_{s}$ is the fiber corresponding to a scrollar syzygy $s$.

Now consider the incidence variety

$$
I=\left\{(F, c) \mid c \in F \cap C \subset \mathbb{P}^{g-1}\right\} \subset \mathbb{G} \times C
$$

and the diagram

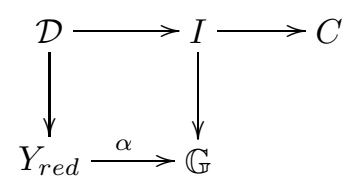

obtained by base change. $\mathcal{D}$ is a family of divisors. The fiber over a scrollar syzygy $s$ is the divisor $D_{s}$ cut out by the fiber $F_{s}$ corresponding to $s$. Lemma 6.8 shows that these divisors all have degree $d=\left\lceil\frac{g+2}{2}\right\rceil$ and $r=1$. By the universal property of $C_{d}^{r}$ we obtain a morphism

$$
\zeta: Y_{\text {red }} \rightarrow C_{\left\lceil\frac{g+2}{2}\right\rceil}^{1} .
$$

To prove the surjectivity of $\zeta$ let $D \in C_{\left\lceil\frac{g+2}{2}\right\rceil}^{1}$ be any divisor. The scroll $S_{|D|}$ spanned by $|D|$ has codimension $g-\left\lceil\frac{g+2}{2}\right\rceil$ by Proposition 6.1, and the fiber $\operatorname{span} D$ corresponds to a scrollar $\left\lceil\frac{g-5}{2}\right\rceil$ th (last) syzygy $s$ with $F_{s}=\operatorname{span} D$ as in Example 4.4. This implies $D \subset F_{s} . C$. Equality follows since they have the same degree by Lemma 6.8

We are left to prove that $\zeta$ is injective. Assume $s, t$ are two last scrollar syzygies, whose fibers $F_{s}$ and $F_{t}$ in their respective scrolls $S_{s}$ and $S_{t}$ cut out the same divisor $D=F_{s} . C=F_{t} . C$. Then the scroll $S_{|D|}$ obtained from the complete pencil $|D|$ is contained in $S_{s}$ and $S_{t}$. Now all these scrolls are of the same dimension, so they 
have to be equal. Since there is a $1: 1$ correspondence between divisors $D^{\prime} \in|D|$, fibers span $D^{\prime}$ and scrollar syzygies of $S_{|D|}=S_{s}=S_{t}$, we must have $s=t$.

So $\zeta$ is bijective. Now $Y_{\text {red }}$ is reduced by definition, and since $C$ is a general canonical curve, $C_{\left\lceil\frac{g+2}{2}\right\rceil}^{1}$ is normal. So by Zariski's Main Theorem $\zeta$ is an isomorphism.

The description of $Y_{\text {red }} \cong C_{\left\lceil\frac{g+2}{2}\right\rceil}^{1}$ is obtained from Brill-Noether theory: For a general canonical curve the dimension of $C_{\left\lceil\frac{g+2}{2}\right\rceil}^{1}$ is given by [ACGH85, p. 214]:

$$
\begin{aligned}
\operatorname{dim} C_{\left\lceil\frac{g+2}{2}\right\rceil}^{1} & =\rho+1 \\
& =g-2\left(g-\left\lceil\frac{g+2}{2}\right\rceil+1\right)+1 \\
& =2\left\lceil\frac{g+2}{2}\right\rceil-1-g \\
& = \begin{cases}1 & \text { for } g \text { even, } \\
2 & \text { for } g \text { odd } .\end{cases}
\end{aligned}
$$

Now the Abel-Jacobi map

$$
\begin{aligned}
\alpha: C_{\left\lceil\frac{g+2}{2}\right\rceil}^{1} & \rightarrow W_{\left\lceil\frac{g+2}{2}\right\rceil}^{1} \\
D & \mapsto|D|
\end{aligned}
$$

has $\mathbb{P}^{1}$-fibers, so $C_{\left\lceil\frac{g+2}{2}\right\rceil}^{1}$ is a disjoint union of finitely many $\mathbb{P}^{1}$ 's for $g$ even and a ruled surface over $W_{\left\lceil\frac{g+2}{2}\right\rceil}^{1}$ for $g$ odd.

In the even case the number of $\mathbb{P}^{1}$ 's can be calculated by a formula of Castelnuovo ACGH85, p. 211]:

$$
\operatorname{deg} W_{\left\lceil\frac{g+2}{2}\right\rceil}^{1}=g ! \prod_{i=0}^{1} \frac{i !}{\left(g-\left\lceil\frac{g+2}{2}\right\rceil+1+i\right) !}=\frac{2}{g+2}\left(\begin{array}{c}
g \\
\frac{g}{2}
\end{array}\right) .
$$

Example 6.10. A general canonical curve of genus 8 has 14 complete pencils $\left|D_{i}\right|$ of degree 5. Each such $g_{5}^{1}$ defines a scroll $S_{\left|D_{i}\right|}$ of degree $8-5+1=4$ that contains $C$. The corresponding minimal free resolutions are subcomplexes of the linear strand of $C$ :

$$
\begin{array}{ccccccccc}
1 & - & - & - & \subset & 1 & - & - & - \\
- & 6 & 8 & 3
\end{array} \quad-\quad \begin{array}{lll}
15 & 35 & 21
\end{array}
$$

The last syzygy space of each scroll $S_{\left|D_{i}\right|}$ is a $\mathbb{P}^{2} \cong \mathbb{P}\left(3^{*}\right)$ that contains a conic of rank 4 syzygies that are in 1:1 correspondence with the fibers of $S_{i}$ and the elements of $\left|D_{i}\right|$. So we obtain 14 conics in the last syzygy space $\mathbb{P}\left(21^{*}\right) \cong \mathbb{P}^{20}$ of $C$. We will show later that this configuration of conics is nondegenerate and lies on a 2-uple embedded $\mathbb{P}^{5} \hookrightarrow \mathbb{P}^{20}$ (see Figure 8.3).

\section{Mukai VARIEties}

By a construction of Mukai one can construct general canonical curves of low genus by taking general sections of certain rational homogeneous varieties.

Theorem 7.1 (Mukai). Every general canonical curve of genus $7 \leq g \leq 9$ is a general linear section of an embedded rational homogeneous (Mukai) variety $M_{g}$. 
General canonical curves of genus 6 are cut out by a general quadric on a general linear section of a homogeneous (Mukai) variety $M_{6}$.

More explicitly we have

\begin{tabular}{|c|c|}
\hline$g$ & $M_{g}$ \\
\hline 6 & the Grassmannian $\mathbb{G}(2,5) \subset \mathbb{P}^{9}$ \\
7 & the Spinor-Variety $S_{10}^{+} \subset \mathbb{P}^{15}$ \\
8 & the Grassmannian $\mathbb{G}(2,6) \subset \mathbb{P}^{14}$ \\
9 & the symplectic Grassmannian $\operatorname{Gr}(3,6, \eta) \subset \mathbb{P}^{13}$ \\
\hline
\end{tabular}

Proof. Muk92b], Muk92a].

Our idea is now to use representation theory to calculate the linear strand, the variety of minimal rank syzygies and the vector bundle of linear forms for $M_{6}, M_{7}$ and $M_{8}$, and to then use the restriction methods developed in section 2 to obtain results about general canonical curves.

Let us start with the linear strands.

Proposition 7.2. The linear strand of $\mathbb{G}(V, 2)$ is

$$
F_{\bullet}(2) \rightarrow I_{\mathbb{G}(V, 2)}
$$

with $F_{i 2}=\Lambda_{4+i, 1^{i}} V$, where $\Lambda_{\lambda} V$ denotes the Schur functor of the partition $\lambda$.

The linear strand of $S_{10}^{+}$is

$$
\operatorname{spin}_{5}^{-} \otimes \mathcal{O}(-3) \rightarrow \Lambda_{1} \otimes \mathcal{O}(-2) \rightarrow I_{S_{10}^{+}}
$$

where $\operatorname{spin}_{5}^{-}$and $\Lambda_{1}$ are irreducible representations of $\mathrm{SO}(10)$ of dimension 16 and 10, respectively.

Proof. The ideal of $\mathbb{G}(V, 2)$ is generated by the $4 \times 4$-Pfaffians of the generic skew symmetric matrix

$$
V \otimes V \rightarrow \Lambda^{2} V
$$

Har92, Ex. 9.20]. The minimal free resolution of Pfaffian ideals is calculated by Józefiak, Pragacz and Weyman in [JPW81, Thm 3.14].

The Betti numbers of $S_{10}^{+}$are calculated for example in Muk92a]:

$$
\begin{array}{cccccc}
1 & - & - & - & - & - \\
- & 10 & 16 & - & - & - \\
- & - & - & 16 & 10 & - \\
- & - & - & - & - & 1
\end{array}
$$

This gives the linear strand as

$$
F_{12} \otimes \mathcal{O}(-3) \rightarrow F_{02} \otimes \mathcal{O}(-2) \rightarrow I_{S_{10}^{+}}
$$

with $\operatorname{dim} F_{12}=16$ and $\operatorname{dim} F_{02}=10$. Now $F_{02}$ is an $\mathrm{SO}(10)$ invariant subset of quadrics in $\mathbb{P}\left(\operatorname{spin}_{5}^{+}\right)$:

$$
F_{02} \subset S_{2}\left(\operatorname{spin}_{5}^{+}\right) \subset \operatorname{spin}_{5}^{+} \otimes \operatorname{spin}_{5}^{+}=\Lambda_{5}^{+} \oplus \Lambda_{3} \oplus \Lambda_{1},
$$

where $\Lambda_{5}^{+}$is the irreducible representation corresponding to the maximal weight vector $L_{1}+\cdots+L_{5}$. The representations have dimension 126, 120 and 10, respectively. Therefore $F_{02}=\Lambda_{1}$. (For the decomposition of the tensor products see KN88.)

In the next step we know

$$
F_{12} \subset \Lambda_{1} \otimes \operatorname{spin}_{5}^{+}=\lambda_{1} \cdot \operatorname{spin}_{5}^{+} \oplus \operatorname{spin}_{5}^{-}
$$


where $\lambda_{1} \cdot \operatorname{spin}_{5}^{+}$denotes the irreducible representation obtained by adding the maximal weights of $\Lambda_{1}$ and $\operatorname{spin}_{5}^{+}$. The irreducible summands have dimensions 144 and 16 so that $F_{12}$ must be equal to $\operatorname{spin}_{5}^{-}$.

Representation theory also helps in determining the spaces of minimal rank last syzygies.

Proposition 7.3. For $\mathbb{G}(V, 2)$ we have

$$
\mathbb{P}\left(V^{*}\right) \hookrightarrow Y_{\min } \subset \mathbb{P}\left(\Lambda_{v, 1^{v-4}} V^{*}\right) \cong \mathbb{P}\left(S_{v-4} V^{*}\right)
$$

where $v=\operatorname{dim} V$ and the inclusion is the $(v-4)$-uple embedding.

For $S_{10}^{+}$we have

$$
S_{10}^{+} \hookrightarrow Y_{\min } \subset \mathbb{P}\left(\left(\operatorname{spin}_{5}^{-}\right)^{*}\right)
$$

Proof. The rank of a last syzygy $s \in \mathbb{P}\left(\Lambda_{v, 1^{v-4}} V^{*}\right)$ is invariant under the action of $\mathrm{GL}(V)$. Consequently $Y_{\min }$ is also invariant. Since $Y_{\min }$ is compact and $\Lambda_{v, 1^{v-4}} V$ is an irreducible $\mathrm{GL}(V)$-representation, $Y_{\min }$ has to contain the minimal orbit of $\mathrm{GL}(V)$ in $\mathbb{P}\left(\Lambda_{v, 1^{v-4}} V^{*}\right) \cong \mathbb{P}\left(S_{v-4} V^{*}\right)$. This is the $(v-4)$-uple embedding of $\mathbb{P}\left(V^{*}\right)$.

A similar line of reasoning gives the result for $S_{10}^{+}$.

The last result in this section determines the vector bundle of linear forms $L_{\text {min }}$ on the minimal orbits of the above proposition.

Proposition 7.4. Let $L_{\text {min }}$ be the vector bundle of linear forms on the space of last minimal rank syzygies $Y_{\min } \subset \mathbb{P}\left(\Lambda_{v, 1^{v-4}} V^{*}\right)$ of $\mathbb{G}(V, 2)$. Then we have

$$
L_{\text {min }} \otimes \mathcal{O}_{\mathbb{P}\left(V^{*}\right)}=\mathcal{T}_{\mathbb{P}\left(V^{*}\right)}(-2)
$$

where $\mathbb{P}\left(V^{*}\right)$ is the minimal orbit of $\mathrm{GL}(V)$ in $\mathbb{P}\left(\Lambda_{v, 1^{v-4}} V^{*}\right)$.

If $L_{\text {min }}$ is the vector bundle of linear forms on the space of last minimal rank syzygies $Y_{\min } \subset \mathbb{P}\left(\left(\operatorname{spin}_{5}^{-}\right)^{*}\right)$ of $S_{10}^{+}$, then

$$
L_{\min } \otimes \mathcal{O}_{S_{10}^{+}}=\mathcal{B}(-1),
$$

where $\mathcal{B}$ is the universal quotient bundle on $S_{10}^{+}$.

Proof. Consider the last triple tensor of the linear strand of $\mathbb{G}(V, 2)$ :

$$
\gamma: \Lambda_{v, 1^{v-4}} V \otimes\left(\Lambda_{v-1,1^{v-5}} V\right)^{*} \rightarrow \Lambda^{2} V .
$$

Let $s \in \mathbb{P}\left(V^{*}\right) \hookrightarrow \mathbb{P}\left(\Lambda_{v, 1^{v-4}} V^{*}\right)$ be a syzygy in the minimal orbit. After choosing a suitable basis $\left\{v_{1}, \ldots, v_{v}\right\}$ we can assume that $s=v_{1}$ and its image in $\mathbb{P}\left(\Lambda_{v, 1^{v-4}} V^{*}\right)$ is represented by the Young tableau of minimal weight

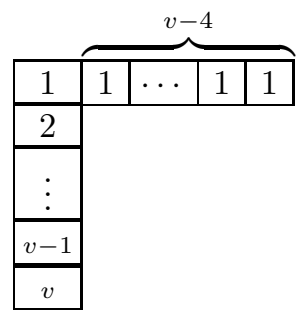


The Littlewood-Richardson decomposition

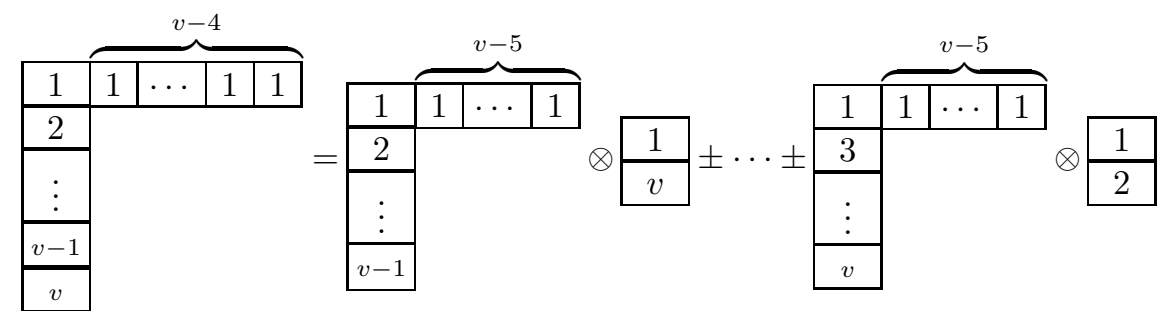

then shows that the image of

$$
\gamma_{\Lambda_{v, 1} 1^{v-4} V^{*}}: \mathcal{O}(-1) \otimes\left(\Lambda_{v-1,1^{v-5}} V\right)^{*} \rightarrow \Lambda^{2} V
$$

over $s=v_{1}$ is $\left\langle v_{1} \wedge v_{v}, \ldots, v_{1} \wedge v_{2}\right\rangle=v_{1} \wedge V$. On $\mathbb{P}\left(V^{*}\right)$ we therefore have a diagram

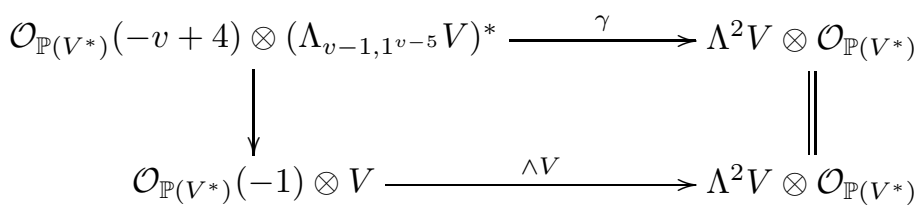

Now by the definition of $L_{\min }$ the image of the top map is $L_{\min } \otimes \mathcal{O}_{\mathbb{P}\left(V^{*}\right)}$. The image of the lower map is $\mathcal{T}_{\mathbb{P}\left(V^{*}\right)}(-2)$ since its kernel is $\mathcal{O}(-2)$. This proves the first claim. The second claim follows by a slightly more complicated reasoning using the representation theory of $\mathrm{SO}(10)$.

\section{Genus 8}

In this section we will describe the spaces of last scrollar syzygies for general canonical curves of genus 8 .

Consider a general canonical curve $C \subset \mathbb{P}^{7}=\mathbb{P}(W)$ of genus $g=8$. By Mukai's Theorem there is an embedding of $\mathbb{P}^{7} \subset \mathbb{P}^{14}=\mathbb{P}\left(\Lambda^{2} V\right)$ given by a projection

$$
\Lambda^{2} V \rightarrow W
$$

such that

$$
C=\mathbb{G}(6,2) \cap \mathbb{P}^{7} .
$$

Since this intersection is of expected codimension and $\mathbb{G}(6,2)$ is ACM, $C$ will have the same Betti numbers as $\mathbb{G}(6,2)$. In particular its linear strand will be

$$
\Lambda_{611} V \otimes \mathcal{O}(-4) \rightarrow \Lambda_{51} V \otimes \mathcal{O}(-3) \rightarrow \Lambda_{4} V \otimes \mathcal{O}(-2) \rightarrow I_{C}
$$

with the last differential given by the restricted triple tensor

$$
\Lambda_{611} V \otimes\left(\Lambda_{51} V\right)^{*} \stackrel{\gamma}{\longrightarrow} \Lambda^{2} V \longrightarrow W .
$$

So we can use the vector bundle of linear forms $L_{\min }=\mathcal{T}_{\mathbb{P}^{5}}(-2)$ on the minimal orbit $\mathbb{P}^{5} \subset \mathbb{P}\left(\Lambda_{611} V^{*}\right)=\mathbb{P}^{20}$ to calculate the restricted ranks of syzygies in the minimal orbit.

Proposition 8.1. Consider the map of vector bundles

$$
\iota^{\prime}: \mathcal{T}_{\mathbb{P}^{5}}(-2) \rightarrow \Lambda^{2} V \otimes \mathcal{O}_{\mathbb{P}^{5}} \rightarrow W \otimes \mathcal{O}_{\mathbb{P}^{5}}
$$


on the minimal orbit $\mathbb{P}^{5} \hookrightarrow \mathbb{P}^{20}$ of $\mathrm{GL}(6)$ in the space of last linear syzygies of $C$. Then the rank 4 locus $Z_{4}\left(i^{\prime}\right)$ is reduced and of expected dimension 1 . It is contained in the space of scrollar last syzygies of $C$.

Proof. Since $\mathcal{T}_{\mathbb{P}^{5}}(-2)^{*}$ is globally generated by $\Lambda^{2} V=H^{0}\left(\mathcal{T}_{\mathbb{P}^{5}}(-2)^{*}\right)$ and the projection $\Lambda^{2} V \rightarrow W$ is general, the determinantal scheme $Z_{4}\left(\iota^{\prime}\right)$ is reduced and of expected dimension

$$
\operatorname{dim} Z_{4}\left(\iota^{\prime}\right) \geq 5-\left(\operatorname{rank} \mathcal{T}_{\mathbb{P}^{5}}-4\right)(\operatorname{rank} W-4)=1 .
$$

All syzygies in $Z_{4}\left(i^{\prime}\right)$ are 2 nd syzygies of restricted rank 4 , i.e. by definition are last scrollar syzygies of $C$.

Corollary 8.2. $Z_{4}\left(\iota^{\prime}\right)$ is the space of last scrollar syzygies of $C$, i.e. a union of 14 skew conics on the 2-uple embedding of $\mathbb{P}^{5}$ in $\mathbb{P}^{20}$.

Proof. By Corollary 6.9 the space of scrollar last syzygies of $C$ is isomorphic to

$$
C_{5}^{1}=\bigcup_{i=1}^{14} \mathbb{P}^{1}
$$

This shows that $Z_{4}\left(\iota^{\prime}\right)$ is the union of at most 14 disjoint $\mathbb{P}^{1}$ 's. Each of these $\mathbb{P}^{1}$ is the scheme of second minimal rank syzygies of a scroll. These schemes are rational normal curves of degree 2 as calculated in Example 4.4. Since they lie on the 2-uple embedding of $\mathbb{P}^{5}$ in $\mathbb{P}^{20}$ they are the images of lines in $\mathbb{P}^{5}$.

Now $Z_{4}\left(\iota^{\prime}\right)$ is of expected codimension, and we can calculate its class using the Porteous formula [ACGH85, p. 86]:

$$
z_{4}\left(\iota^{\prime}\right)=\Delta_{8-4,5-4}\left(\frac{c_{t}\left(8 \mathcal{O}_{\mathbb{P}^{5}}\right)}{c_{t}\left(\mathcal{I}_{\mathbb{P}^{5}}(-2)\right)}\right)=14 H^{3},
$$

i.e. $z_{4}\left(\iota^{\prime}\right)$ is the class of 14 lines in $\mathbb{P}^{5}$. Since $Z_{4}\left(\iota^{\prime}\right)$ is reduced, the desired equality follows.

From this we can finally prove the scrollar syzygy conjecture for the last syzygies of $C$.

Theorem A. The scheme $Z$ of last scrollar syzygies of a general canonical curve $C \subset \mathbb{P}^{7}$ of genus 8 is a configuration of 14 skew conics that lie on a 2 -uple embedded $\mathbb{P}^{5} \hookrightarrow \mathbb{P}^{20}$. $Z$ spans the whole $\mathbb{P}^{20}$ of second syzygies of $C$.

Proof. We have to prove that $Z=Z_{4}\left(\iota^{\prime}\right)$ does not lie on any quadric. Since $Z_{4}\left(\iota^{\prime}\right)$ is the determinantal locus where $\iota^{\prime}$ drops rank by 1 , its ideal is resolved by the Eagon-Northcott complex

$$
E_{\bullet} \rightarrow I_{Z_{4}\left(\iota^{\prime}\right)}
$$

with

$$
E_{i}=\Lambda^{5+k} W^{*} \otimes S_{i}\left(\mathcal{T}_{\mathbb{P}^{5}}(-2)\right)=\left(\begin{array}{l}
8 \\
k
\end{array}\right) S_{i}\left(\mathcal{T}_{\mathbb{P}^{5}}(-2)\right) .
$$

Using the theorem of Bott Ott95, Theorem 11.4] one finds

$$
H^{j}\left(E_{i}(2)\right)=0
$$

for all $i, j$ except for $(i, j)=(3,4)$. By a diagram chase this implies

$$
H^{j}\left(I_{Z_{4}\left(\iota^{\prime}\right)}(2)\right)=0
$$

for $j \neq 1$. In particular there exists no quadric that contains $Z_{4}\left(\iota^{\prime}\right)$. 


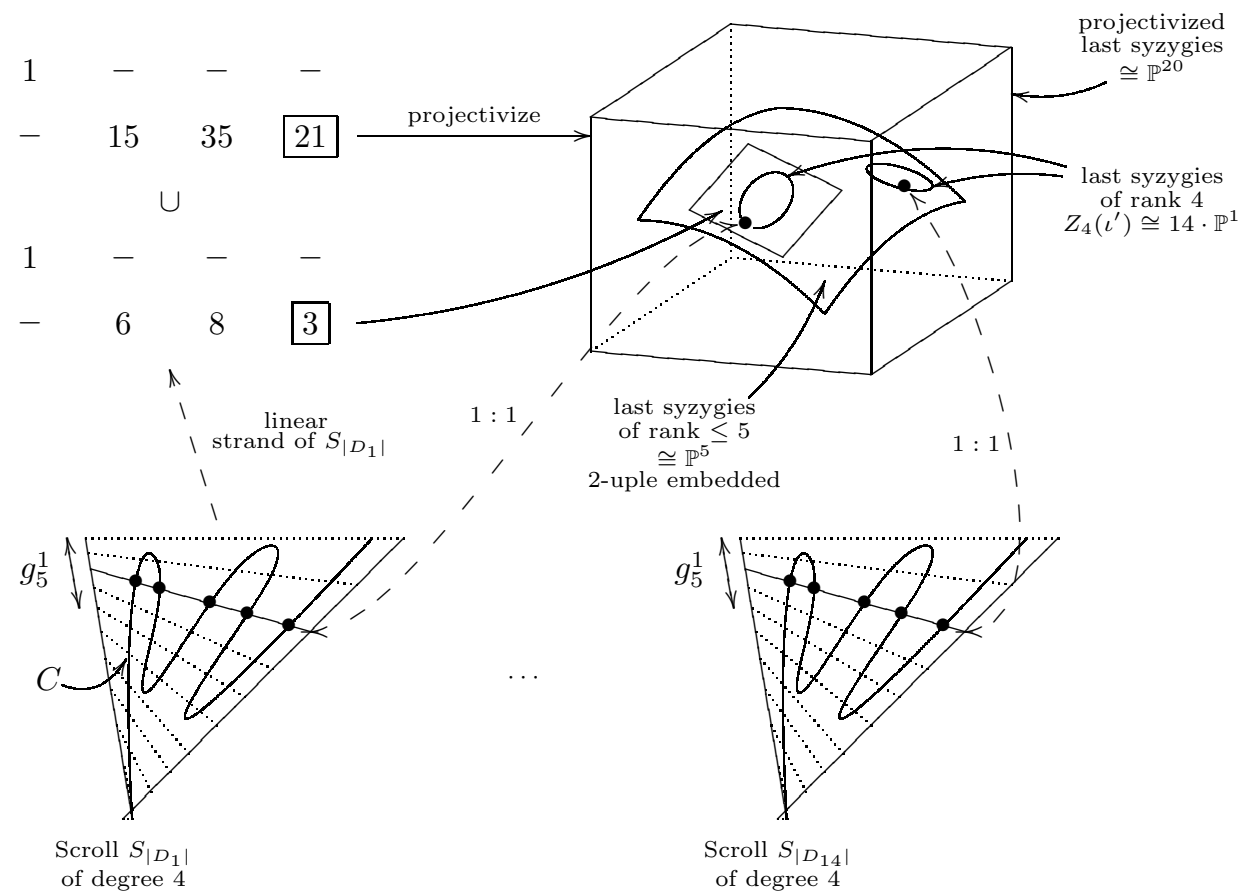

Figure 8.3. A general canonical curve of genus 8 lies on 14 scrolls of degree 4 . These scrolls define 14 pencils of degree 5 and 14 conics of rank 4 syzygies. These conics all lie on a 2 -uple embedded $\mathbb{P}^{5}$ of rank $\leq 5$ syzygies.

Remark 8.4. A calculation similar to the one above is also contained in Voi02, but with a different aim.

Remark 8.5. Note that $H^{1}\left(I_{Z_{4}\left(\iota^{\prime}\right)}\right) \neq 0$. In particular the scheme of last scrollar syzygies is not linearly normal.

\section{Genus 7}

In this section we describe the space of scrollar syzygies of general canonical curves $C \subset \mathbb{P}^{6}$ of genus 7 . Since the reasoning is quite similar to the last section we only sketch the proofs and leave the details to the reader.

In this case Mukai's Theorem implies that the linear strand of $C$ is

$$
\operatorname{spin}_{5}^{-} \otimes \mathcal{O}(-3) \rightarrow \Lambda_{1} \otimes \mathcal{O}(-2) \rightarrow I_{C}
$$

with the last differential given by a restricted triple tensor

$$
\operatorname{spin}_{5}^{-} \otimes\left(\Lambda_{1}\right)^{*} \stackrel{\gamma}{\longrightarrow} \operatorname{spin}_{5}^{+} \longrightarrow W
$$

with $\mathbb{P}^{6}=\mathbb{P}(W)$.

Proposition 9.1. Consider the map of vector bundles

$$
\iota^{\prime}: \mathcal{B}(-1) \rightarrow \operatorname{spin}_{5}^{+} \otimes \mathcal{O} \rightarrow W \otimes \mathcal{O}
$$


on the minimal orbit $S_{10}^{+} \hookrightarrow \mathbb{P}^{15}$ of $\mathrm{SO}(10)$ in the space of last linear syzygies of $C$. Then the rank 3 locus $Z_{3}\left(i^{\prime}\right)$ is the space of scrollar last syzygies of $C$.

Proof. Since $\mathcal{B}(-1)^{*}$ is globally generated and the projection $\operatorname{spin}_{5}^{+} \rightarrow W$ is general, the determinantal scheme $Z_{3}\left(\iota^{\prime}\right)$ is reduced and of expected dimension

$$
\operatorname{dim} Z_{3}\left(\iota^{\prime}\right) \geq 5-(\operatorname{rank} \mathcal{B}-3)(\operatorname{rank} W-3)=2 .
$$

All syzygies in $Z_{3}\left(i^{\prime}\right)$ are first syzygies of restricted rank 3, i.e. by definition are last scrollar syzygies of $C$. Since the space of scrollar last syzygies in this case is isomorphic to the ruled surface $C_{5}^{1}$ which is in particular reduced and irreducible, the two spaces are the same.

Theorem B. The scheme $Z$ of last scrollar syzygies of a general canonical curve $C \subset \mathbb{P}^{6}$ of genus 7 is a linearly normal ruled surface of degree 84 on a spinor variety $S_{10}^{+} \subset \mathbb{P}^{15}$. This ruled surface spans the whole $\mathbb{P}^{15}$ of first syzygies of $C$.

Proof. $Z=Z_{3}\left(\iota^{\prime}\right)$ is a ruled surface, since it is isomorphic to $C_{5}^{1}$. By the Porteous formula the class of $Z_{3}\left(\iota^{\prime}\right)$ in $S_{10}^{+}$is $7 H^{8}$. Since the degree of $S_{10}^{+}$is 12 , the degree of $Z_{3}\left(\iota^{\prime}\right)$ is 84 .

To show that $Z_{3}\left(\iota^{\prime}\right)$ is nondegenerate, we have to show that $I_{Z_{3}\left(\iota^{\prime}\right)}$ contains no elements of degree 1 . Since $Z_{3}\left(\iota^{\prime}\right)$ is the locus where $\iota^{\prime}$ drops rank by 2 , the resolution of its ideal sheaf can be calculated by the methods of Lascoux Las78, Thm 3.3] to be:

$$
\begin{aligned}
0 & \rightarrow \Lambda_{77} \otimes S_{44222} \rightarrow \Lambda_{76} \otimes S_{43222} \\
& \rightarrow \Lambda_{75} \otimes S_{42222}+\Lambda_{66} \otimes S_{33222} \\
& \rightarrow \Lambda_{71} \otimes S_{41111}+\Lambda_{65} \otimes S_{32222} \\
& \rightarrow \Lambda_{7} \otimes S_{4111}+\Lambda_{61} \otimes S_{31111}+\Lambda_{55} \otimes S_{22222} \\
& \rightarrow \Lambda_{6} \otimes S_{3111}+\Lambda_{51} \otimes S_{21111} \\
& \rightarrow \Lambda_{5} \otimes S_{2111}+\Lambda_{41} \otimes S_{11111} \rightarrow \Lambda_{4} \otimes S_{1111} \rightarrow I_{Z_{3}\left(\iota^{\prime}\right)},
\end{aligned}
$$

where $\Lambda_{\lambda}=\Lambda_{\lambda} W^{*}$ and $S_{\mu}=S_{\mu}(\mathcal{B}(-1)$ ). (This is resolution $k(\alpha, 2,0)$ in the notation of Lascoux.) Again using the theorem of Bott and a diagram chase one calculates that

$$
H^{j}\left(I_{Z_{3}\left(\iota^{\prime}\right)}(1)\right)=0
$$

for $j \neq 2$. In particular $Z_{3}\left(\iota^{\prime}\right)$ is nondegenerate and linearly normal.

Remark 9.2. The degree of this ruled surface can also be obtained via Brill-Noether theory vB00, p. 54, Satz 5.1].

\section{Genus 6}

In this section we describe the last scrollar syzygies of a general curve of genus 6. This is well known; we only include it because it also follows from our methods.

By Mukai's Theorem we obtain a general canonical curve $C \subset \mathbb{P}^{5}=\mathbb{P}(W)$ of genus 6 by a general intersection

$$
C=\mathbb{G}(5,2) \cap \mathbb{P}^{5} \cap Q
$$

where $Q$ is a general quadric in $\mathbb{P}^{5}$. As above $\mathbb{G}(5,2) \cap \mathbb{P}^{5}$ has the same Betti numbers as $\mathbb{G}(5,2)$, in particular a linear strand

$$
\Lambda_{51} V \otimes \mathcal{O}(-3) \rightarrow \Lambda_{4} V \otimes \mathcal{O}(-2) \rightarrow I_{\mathbb{G}(5,2) \cap \mathbb{P}^{5}}
$$


with the last differential given by the restricted triple tensor

$$
\Lambda_{611} V \otimes\left(\Lambda_{51} V\right)^{*} \stackrel{\gamma}{\longrightarrow} \Lambda^{2} V \longrightarrow W .
$$

Since $Q$ intersects $\mathbb{G}(5,2) \cap \mathbb{P}^{5}$ transversally the linear strand of $C$ is

$$
\Lambda_{51} V \otimes \mathcal{O}(-3) \rightarrow\left(\Lambda_{4} V \oplus \mathbb{C}\right) \otimes \mathcal{O}(-2) \rightarrow I_{C},
$$

in particular the last differential is still given by the same triple tensor. From this a completely analogous reasoning as in the case $g=8$ gives

Theorem C. The scheme $Z$ of last scrollar syzygies of a general canonical curve $C \subset \mathbb{P}^{5}$ of genus 6 is a configuration of 5 skew lines in $\mathbb{P}^{4}$ that spans the whole $\mathbb{P}^{4}$ of first syzygies of $C$.

Remark 10.1. The space of last scrollar syzygies is again not linearly normal in this case.

\section{REFERENCES}

[ACGH85] E. Arbarello, M. Cornalba, P.A. Griffiths, and J. Harris. Geometry of Algebraic Curves. Grundlehren der mathematischen Wissenschaften 129. Springer, Heidelberg, 1985. MR0770932 (86h:14019)

[AH81] E. Arbarello and J. Harris. Canonical Curves and Quadrics of Rank 4. Comp. Math., 43:145-179, 1981. MR0622446 (82k:14020)

[AM67] A. Andreotti and A. Mayer. On period relations for abelian integrals on algebraic curves. Ann. Scuola Norm. Sup. Pisa, 21:189-238, 1967. MR0220740(36:3792)

[Ehb94] S. Ehbauer. Syzygies of points in projective space and applications. In F. Orecchia, editor, Zero-dimensional schemes. Proceedings of the international conference held in Ravello, Italy, June 8-13, 1992, pages 145-170, Berlin, 1994. de Gruyter. MR 1292482 (95h:13012)

[Eis88] D. Eisenbud. Linear sections of determinantal varieties. Amer. J. Math., 110(3):541575, 1988. MR0944327 (89h:14041)

[GL84] M. Green and R. Lazarsfeld. The non-vanishing of certain Koszul cohomology groups. J. Diff. Geom., 19:168-170, 1984.

[Gre84a] M.L. Green. Koszul cohomology and the geometry of projective varieties. J. Differential Geometry, 19:125-171, 1984. MR0739785 (85e:14022)

[Gre84b] M.L. Green. Quadrics of rank four in the ideal of a canonical curve. Inv. Math., 75:85104, 1984. MR0728141 (85f:14028)

[GS02] Daniel R. Grayson and Michael E. Stillman. Macaulay 2, a software system for research in algebraic geometry. Available at http://www.math.uiuc.edu/Macaulay2, 2002.

[Har92] J. Harris. Algebraic Geometry. Graduate Texts in Math. 133. Springer, Heidelberg, 1992. MR 1182558 (93j:14001)

[HR98] A. Hirschowitz and S. Ramanan. New evidence for Green's conjecture on syzygies of canonical curves. Ann. Sci. Ec. Norm. Super., IV Ser 31(4):145-152, 1998. MR.1603255 (99a:14033)

[JPW81] T. Józefiak, P. Pragacz, and J. Weyman. Resolutions of determinantal varieties and tensor complexes associated with symmetric and antisymmetric matrices, 1981.

[KN88] G. Kempf and L. Ness. Tensor products of fundamental representations. Can. J. Math, XL(3):633-648, 1988. MR0960599 (89m:20046)

[Las78] A. Lascoux. Syzygis des variétés déterminantales. Adv. Math., 30:202-237, 1978. MR 0520233 (80j:14043)

[Muk92a] S. Mukai. Curves and symmetric spaces. Proc. Japan Acad. Ser. A Math. Sci., 68:7-10, 1992. MR1158012 (93d:14042)

[Muk92b] S. Mukai. Fano 3-folds. In G. Ellingsrud, C. Peskine, G. Sacchiero, and S.A. Stromme, editors, Complex Projective Geometry, volume 179 of LMS Lecture Note Series, pages 255-261. Cambridge University Press, 1992. MR.1201387 (94a:14042) 
[Ott95] G. Ottaviani. Rational Homogeneous Varieties. Lecture notes for the summer school in Algebraic Geometry in Cortona, 1995.

[Pet23] K. Petri. Über die invariante Darstellung algebraischer Funktionen einer Veränderlichen. Math. Ann., 88:242-289, 1923. MR.1512130

[Sch86] F.O. Schreyer. Syzygies of canonical curves and special linear series. Math. Ann., 275:105-137, 1986. MR0849058 (87j:14052)

[Sch88] F.O. Schreyer. Green's Conjecture for general p-gonal Curves of large Genus. In E. Ballico and C. Ciliberto, editors, Algebraic Curves and Projective Geometry, volume 1389 of Lecture Notes in Mathematics, pages 254-260, Springer, Heidelberg, 1988. MR.1023403 (90j:14041)

[Sch91] F.O. Schreyer. A standard basis approach to syzygies of canonical curves. J. Reine Angew. Math., 421:83-123, 1991. MR.1129577 (92j:14040)

[TiB02] Montserrat Teixidor i Bigas. Green's conjecture for the generic $r$-gonal curve of genus $g \geq 3 r-7$. Duke Math. J., 111(2):195-222, 2002. MR.1882133 (2003a:14046)

[vB00] H.-Chr. Graf v. Bothmer. Geometrische Syzygien von kanonischen Kurven. Dissertation, Universität Bayreuth, 2000.

[Voi88] C. Voisin. Courbes tétragonal et cohomologie de Koszul. J. Reine Angew. Math., 387:111-121, 1988. MR0946352 (89e:14036)

[Voi02] C. Voisin. Green's generic syzygy conjecture for curves of even genus lying on a $K 3$ surface. J. Eur. Math. Soc. (JEMS), 4(4):363-404, 2002. MR.1941089(2003i:14040)

[Voi05] Claire Voisin. Green's canonical syzygy conjecture for generic curves of odd genus. Compos. Math., 141(5):1163-1190, 2005. MR.2157134(2006c:14053)

Laboratoire J.-A. Dieudonné, Université de Nice, Parc Valrose, 06108 Nice cedex 2, FRANCE

Current address: Institiut für Algebraische Geometrie, Universität Hannover, Welfengarten 1, D-30167 Hannover, Germany

E-mail address: bothmer@math.uni-hannover.de

URL: http://www-iag.math.uni-hannover.de/ bothmer 